\title{
Prognostic Impact of Acute Kidney Injury on Decompensated Heart Failure
}

\author{
Layane Bonfante Batista, ${ }^{1,}$, Roberto Ramos Barbosa ${ }^{1,2}$, Caroline Feu Rosa Carrera ${ }^{1}$, \\ Gabriella Martins Curcio", Pietro Dall'Orto Lima², Vinicius Angelo Astolpho², \\ Rodolfo Costa Sylvestre ${ }^{2}$, Lucas Crespo De Barros ${ }^{2}$, Renato Giestas Serpa ${ }^{1,2}$, Osmar Araujo Calil ${ }^{1,2}$, \\ Luiz Fernando Machado Barbosa ${ }^{1,2}$
}

${ }^{1}$ Cardiology Department, Holy House of Mercy Medical School, Vitória, Brazil

${ }^{2}$ Cardiology Department, Holy House of Mercy Hospital, Vitória, Brazil

Email address:

bblayane@gmail.com (L. B. Batista), roberto.cardio@gmail.com (R. R. Barbosa), carolfeurosacarrera@gmail.com (C. F. R. Carrera), gabriellamcurcio@gmail.com (G. M. Curcio), pietrolima@gmail.com (P. D. Lima), v.astolpho@gmail.com (V. A. Astolpho), rodolfosylvestre_1993@hotmail.com (R. C. Sylvestre), lucascrespodebarros88@gmail.com (L. C. De Barros), jrserpa@terra.com.br (R. G. Serpa), osmarcalil@uol.com.br(O. A. Calil), uip@terra.com.br (L. F. M. Barbosa) ${ }^{*}$ Corresponding author

\section{To cite this article:}

Layane Bonfante Batista, Roberto Ramos Barbosa, Caroline Feu Rosa Carrera, Gabriella Martins Curcio, Pietro Dall'Orto Lima, Vinicius Angelo Astolpho, Rodolfo Costa Sylvestre, Lucas Crespo De Barros, Renato Giestas Serpa, Osmar Araujo Calil, Luiz Fernando Machado Barbosa. Prognostic Impact of Acute Kidney Injury on Decompensated Heart Failure. American Journal of Internal Medicine. Vol. 9, No. 3, 2021, pp. 121-126. doi: 10.11648/j.ajim.20210903.13

Received: February 28, 2021; Accepted: March 22, 2021; Published: May 26, 2021

\begin{abstract}
Decompensated heart failure (HF) is a complex and debilitating syndrome, which constitutes a severe emergency condition with high morbidity and mortality. Kidneys play fundamental roles in the pathophysiology of HF and, in the context of decompensations, acute kidney injury (AKI) has a bilateral cause-and-effect relationship, which can significantly worsen prognosis. However, the interaction between AKI and decompensated HF is poorly understood. This study aimed to assess the occurrence of AKI in patients hospitalized due to decompensated HF and to analyze its prognostic impact during hospitalization. This prospective single-center observational study included patients hospitalized due to decompensated HF in a tertiary-level teaching hospital, between July 2017 and January 2020. Patients who developed AKI during hospitalization were compared with those who did not develop it, until hospital discharge or death. AKI was defined as a serum creatinine increase greater than or equal to $0.3 \mathrm{mg} / \mathrm{dl}$ in 48 hours, a 1.5 -fold increase in baseline creatinine in seven days or urinary volume $<0.5 \mathrm{ml} / \mathrm{kg} / \mathrm{h}$ for six hours, according to the Acute Kidney Injury Network (AKIN) criteria. The endpoints analyzed were death, need for invasive mechanical ventilation (IMV), and length of hospital stay. The Wilcoxon, Mann-Whitney and unpaired student $t$ tests were used. Ninety-nine patients were included, with a mean age of $65.4 \pm 14$ years, of which $47(47.5 \%)$ were male and $52(52.5 \%)$ were female. Reduced ejection fraction (EF) was observed in $77.8 \%$ of patients, whilst $22.2 \%$ had a diagnosis of HF with preserved EF. Decompensation clinical classifications were dry and warm $=7(7.1 \%)$, wet and warm $=72(72.7 \%)$, wet and cold $=15(15.1 \%)$ and dry and cold $=5(5.1 \%)$. The average left ventricular ejection fraction was $38.3 \% \pm 15$. AKI occurred in 22 patients $(22.2 \%)$. Comparison between patients who evolved with and without AKI showed higher mortality $(36.4 \%$ vs $10.4 \%, \mathrm{p}=0.004)$ and need for IMV (54.5\% vs $13 \%$, $\mathrm{p}=0.0001)$ in the first group. There was no significant difference regarding the length of hospitalization $(22.9 \pm 19$ vs $18.8 \pm 16$ days, $p=0.26$ ). Our results pointed to the occurrence of AKI was frequent in patients with decompensated HF requiring hospitalization, affecting approximately one out of five patients. This complication was significantly associated with increased mortality and the need for IMV during hospitalization.
\end{abstract}

Keywords: Heart Failure, Acute Kidney Injury, Prognosis, Mortality 


\section{Introduction}

Heart failure (HF) is a complex, debilitating syndrome [1] and affects almost all organs and systems [2]. Across the world, HF affects more than 26 million people, directly reflecting on lower quality of life, high mortality, and high cost for health systems [3]. Despite the development of drugs and effective devices for the treatment of chronic HF, episodes of decompensation that require hospitalization are still very frequent and represent an unfavorable course of the disease [4]. In Brazil, in 2019, decompensated HF was responsible for 187.117 hospital admissions and 21.142 deaths, according to data from DATASUS [5].

The kidneys play fundamental roles in the pathophysiology of HF, and patients affected with this pathology often have abnormalities of renal function. Heartkidney interaction, in which acute or chronic dysfunction of one organ can induce acute or chronic dysfunction of the other, is defined as Cardiorenal Syndrome (SCR), which is classified into five types. Type 1 reflects an acute worsening of cardiac function, leading to acute kidney injury. Type 2 is characterized by the development of chronic kidney disease (CKD) secondary to the constant damage caused by chronic HF. Type 3, on the other hand, consists of an acute worsening of renal function, causing acute cardiac dysfunction, while type 4 refers to CKD contributing to the decrease in cardiac function, cardiac hypertrophy, and/or increasing the risk of adverse cardiac events. Type 5 is the existence of simultaneous renal and cardiac damage because of a systemic disease [6].

The presence of acute kidney injury (AKI) in decompensated HF (type $1 \mathrm{SCR}$ ) is very common. This syndrome is the result of several mechanisms, associated with venous congestion and hemodynamic changes, which result in elevated central venous pressure, tubular and glomerular damage, salt and water retention, activation of the renin-angiotensin-aldosterone system and the sympathetic nervous system, and constriction of afferent and efferent arterioles [7]. The prevalence of type $1 \mathrm{SCR}$ in patients hospitalized due to decompensated $\mathrm{HF}$ ranges from $32 \%$ to $40 \%$. The variability of incidence can be attributed to different criteria that define AKI, different populations studied and different diagnostic accuracy of available methods [2]. There are several clinical limitations for the diagnosis of AKI, such as difficulty in measuring urine output, influence of diuretic therapy on urine output, factors that interfere with the elevation of biomarkers, positive water balance in critically ill patients, and scarcity of resources. All these limitations lead to the underdiagnoses of a syndrome that increases mortality and leads to a worse in-hospital prognosis [8].

In the context of underdiagnoses and the fact that patients with severe renal dysfunction are hardly included in clinical trials, little is known about the interaction between AKI and decompensated HF in the Brazilian reality [1]. Thus, this study aimed to assess the occurrence of AKI in patients hospitalized due to decompensated HF and its prognostic impact during hospitalization.

\section{Methods}

\subsection{Study Design}

Prospective single-center observational study.

\subsection{Population}

Patients aged over 18 years, hospitalized with the diagnosis of decompensated HF between July 2017 and January 2020 were included. Inclusion in the study occurred at any time during hospitalization. Patients whose diagnosis of decompensated HF was not the most likely during clinical investigation were excluded. Patients who were on dialysis before hospitalization were also excluded.

\subsection{Data Collection}

All patients were personally approached and included in the study after signing the Informed Consent Form. Data collection was performed from medical records for baseline clinical characteristics, diagnoses, laboratory tests, treatment implemented, clinical evolution and outcomes. Socioeconomic data, such as race and education, were requested directly from the patient or companion. Patients were followed up throughout the hospitalization period, until hospital discharge or death.

\subsection{Variables Analyzed}

The variables collected were: age (in complete years), sex, ethnicity, educational level, marital status, comorbidities (such as arterial hypertension, diabetes mellitus, dyslipidemia, atrial fibrillation, chronic kidney failure), current smoking, prior myocardial infarction, prior percutaneous coronary intervention and/or coronary-artery bypass graft surgery, prior pacemaker and/or cardiac resynchronization therapy and/or implantable cardioverter defibrillator, previous diagnosis of HF, previous follow-up at the HF clinic of the institution, prior diagnosis of $\mathrm{HF}$ with reduced ejection fraction (HFrEF) or HF with preserved ejection fraction (HFpEF), decompensation classification at admission, medications in use before hospitalization (angiotensinconverting enzyme inhibitors or angiotensin II receptor blockers, beta-blockers, aldosterone antagonist, statins and aspirin), left ventricle ejection fraction on transthoracic echocardiogram during hospitalization, and laboratory test values (creatinine and urea in the first 24 hours after admission).

Patients who developed AKI during hospitalization were compared with those who did not develop it. AKI was defined as a serum creatinine increase greater than or equal to $0.3 \mathrm{mg} / \mathrm{dl}$ in 48 hours, a 1.5 -fold increase in baseline creatinine within seven days or urinary volume $<0.5$ 
$\mathrm{ml} / \mathrm{kg} /$ hour for six hours, according to the Acute Kidney Injury Network (AKIN) criteria.

\subsection{Endpoints}

The outcomes considered as primary endpoints were inhospital death, need for invasive mechanical ventilation (IMV), and length of stay (in days).

\subsection{Ethical Aspects}

The study was approved by the Research Ethics Committee of the institution, under number CAAE 86084518.0.0000.5065. All ethical principles of research involving human beings were respected in accordance with the Declaration of Helsinki, and all participants in the research signed the Informed Consent Form. There was no interference in medical care practice by this research.

\section{Statistical Analysis}

Categorical variables were described as absolute and percentage frequency, and continuous variables as mean and standard deviation. The Statistical Package for the Social Sciences (SPSS) software version 20.0 was used for statistical analysis. For comparative analysis, the Wilcoxon, Mann-Whitney and unpaired student $t$ tests were used, adopting a significance level of $5 \%$.

\section{Results}

Ninety-nine patients were included, with mean age $65.4 \pm 14$ years and slight predominance of females $(52.5 \%)$. Hypertension was the most frequent comorbidity (71.7\%). Twenty-one patients $(21.2 \%)$ had prior CKD, and all of them were on conservative therapy. The demographic and baseline clinical characteristics of the studied population are shown in Table 1.

Table 1. Demographic and baseline clinical characteristics of patients with decompensated heart failure requiring hospitalization.

\begin{tabular}{|c|c|}
\hline Baseline demographic and clinical characteristics & $\mathrm{n}=99$ \\
\hline Age (years), mean \pm SD & $65.4 \pm 14$ \\
\hline \multicolumn{2}{|l|}{ Sex } \\
\hline Male, n (\%) & $47(47.5 \%)$ \\
\hline Female, n (\%) & $52(52.5 \%)$ \\
\hline \multicolumn{2}{|l|}{ Ethnicity } \\
\hline Brown, n (\%) & $40(40.4 \%)$ \\
\hline Caucasian, n (\%) & $32(32.3 \%)$ \\
\hline \multicolumn{2}{|l|}{ Marital status } \\
\hline Married, n (\%) & $51(51.5 \%)$ \\
\hline Single or widowed, n (\%) & $42(42.4 \%)$ \\
\hline \multicolumn{2}{|l|}{ Educational level } \\
\hline Incomplete elementary school, n (\%) & $57(57.6 \%)$ \\
\hline Elementary/High School/Higher Education, n (\%) & $41(41.4 \%)$ \\
\hline Hypertension, n (\%) & $71(71.7 \%)$ \\
\hline Dyslipidemia, n (\%) & $22(22.2 \%)$ \\
\hline Atrial fibrillation, $\mathrm{n}(\%)$ & $23(23.2 \%)$ \\
\hline Smoking, n (\%) & $16(16.2 \%)$ \\
\hline \multicolumn{2}{|l|}{ Chronic kidney disease } \\
\hline Conservative, $\mathrm{n}(\%)$ & $21(21.2 \%)$ \\
\hline Dialytic, n (\%) & 0 \\
\hline Previous PCI or CABG surgery, n (\%) & $28(28.3 \%)$ \\
\hline Previous DPM / CRT / ICD, n (\%) & $4(4.0 \%)$ \\
\hline \multicolumn{2}{|l|}{ Medications in previous use } \\
\hline \multicolumn{2}{|l|}{ Beta-blockers } \\
\hline Carvedilol, n (\%) & $46(46.5 \%)$ \\
\hline Metoprolol succinate, n (\%) & $7(7.1 \%)$ \\
\hline Bisoprolol, n (\%) & $6(6.1 \%)$ \\
\hline \multicolumn{2}{|l|}{ ACEI/ARB } \\
\hline Losartan, n (\%) & $36(36.4 \%)$ \\
\hline Valsartan, n (\%) & $10(10.1 \%)$ \\
\hline \multicolumn{2}{|l|}{ Statins } \\
\hline Simvastatin, $\mathrm{n}(\%)$ & $33(33.3 \%)$ \\
\hline Rosuvastatin/Atorvastatin, n (\%) & $4(4.0 \%)$ \\
\hline Aspirin, $\mathrm{n}(\%)$ & $34(24.3 \%)$ \\
\hline Previous diagnosis of $\mathrm{HF}, \mathrm{n}(\%)$ & $55(55.5 \%)$ \\
\hline Follow-up at the HF Clinic, n (\%) & $14(14.1 \%)$ \\
\hline
\end{tabular}

PCI: percutaneous coronary intervention. CABG: coronary-artery bypass graft. DPM: definitive pacemaker. CRT: cardiac resynchronization therapy. ICD: implantable cardioverter defibrillator. ACEI: angiotensin-converting enzyme inhibitors. ARB: angiotensin receptor blockers. HF: heart failure. 
HFrEF (ejection fraction $<50 \%$ ) was observed in 77 patients $(77.8 \%)$, whilst $22(22.2 \%)$ were diagnosed with HFpEF (EF> 50\%). The decompensation classification at admission was dry and warm in seven patients $(7.1 \%)$, wet and warm in 72 patients $(72.7 \%)$, wet and cold in 15 patients $(15.1 \%)$, and dry and cold in 5 patients $(5.1 \%)$. The average EF on transthoracic echocardiogram during hospitalization was $38.3 \% \pm 15$. The average length of stay was $19.6 \pm 16$ days. Sixteen patients died (16.16\%) and the average time until this outcome was $23 \pm 17$ days. The main causes of death were septic and cardiogenic shocks.

AKI occurred in 22 patients (22.2\%). Comparison between patients who evolved with and without AKI showed significant differences of in-hospital mortality and need for IMV (Table 2). There was no statistical difference regarding the length of stay between the two groups. Among patients who developed AKI, nine (40.9\%) had CKD. Of these, three (13.6\%) underwent dialysis therapy during hospitalization.

Table 2. Outcomes in patients with decompensated heart failure with and without acute kidney injury during hospitalization.

\begin{tabular}{llll}
\hline Variables & With AKI (n=22) & Without AKI (n=77) & P \\
\hline In-hospital death, n (\%) & $8(36.4 \%)$ & $8(10.4 \%)$ & 0.004 \\
Length of hospital stay (days), mean \pm SD & $22.9 \pm 18$ & $18.8 \pm 15$ & 0.26 \\
Need for IMV, n (\%) & $12(54.5 \%)$ & $10(13.0 \%)$ & 0.0001 \\
\hline
\end{tabular}

AKI: acute kidney injury. IMV: invasive mechanical ventilation.

\section{Discussion}

Several studies confirm the deterioration of renal function in patients with HF [9-12], but few address the incidence of AKI in the context of HF decompensation in the present Brazilian scenario. In the current study, we assessed the occurrence of AKI in patients with decompensated HF and its association with poor outcomes. Our results showed that AKI was frequent in HF requiring hospitalization, and patients who developed AKI presented excess mortality and higher need for orotracheal intubation and IMV.

As a comparison, the ADHERE study (Acute Decompensated Heart Failure National Registry), which included more than 100,000 patients hospitalized due to decompensated HF, showed that about a third had AKI. This record also showed worse outcomes, including high rates of admission to the intensive care unit, greater need for ventilatory support and higher mortality in these patients [13], corroborating the findings of our study.

It is known that IMV increases the risk of bronchoaspiration, pneumonia, atelectasis and orotracheal injuries, increasing risk and morbidity of critical patients [14]. Ponce et al. demonstrated that mortality in patients with respiratory failure combined with $\mathrm{AKI}$ is 60 to $80 \%$ in the intensive care unit (ICU), whereas in those without AKI it ranges from 30 to $50 \%$ [15]. High mortality associated with pulmonary congestion and IMV reinforces the need for effective prevention of AKI, with careful clinical monitoring, control of the hydroelectrolytic balance, correction of contributing factors such as hypertension, and interruption of triggering factors such as infection and sepsis. It is also essential to identify the precipitating factors of $\mathrm{HF}$ decompensation, especially if reversible, to guide treatment and prevent adverse outcomes, thus avoiding worsening of renal function [16].

Noteworthy, early diagnosis, screening and beginning of specific treatment for decompensated HF are associated with shorter hospital stays [1]. The length of hospital stays, according to Brazilian data, can vary from seven to nine days in patients without $\mathrm{AKI}$, increasing to eight to 20 days in patients with AKI [15]. Prolonged hospitalization in these cases has a positive association with more advanced stages of renal impairment and with higher mortality [17]. We observe, however, a discrepancy between these data and the results of our study, in which average hospital stay was 18 days for patients without AKI and 22 days for patients with AKI. The increase in hospitalization length found in our study is explained by the sum of the length of stay in the ICU and in the ward, whereas in the studies mentioned above only the total time in the ICU was considered.

Among the 22 patients who developed AKI, nine had prior CKD. There is also the possibility that part of the patients who developed AKI were previously undiagnosed with CKD, because HF and CKD often coexist and share risk factors and interact, leading to worse prognosis [18]. It is also known that comorbidities such as hypertension and diabetes (present in approximately three quarters and in half of the population in this study respectively) can further worsen renal function [1]. It is also worth mentioning that the low cardiac output generated by decompensated HF, associated with possible cardiogenic shock, and the use of contrast for cardiovascular procedures in some patients are also possible causal factors for AKI. Such situations demonstrate the multiplicity of factors involved in the genesis of AKI and corroborate even more the complexity in timely identification of renal function deterioration.

Our group has previously conducted a study that showed significant association of serum hemoglobin $<10 \mathrm{mg} / \mathrm{dl}$ within the first 24 hours of hospitalization due to HF with the occurrence of AKI [19]. Although it was not included in the present analysis, the role of anemia should be highlighted as an important marker of HF severity and prognosis. Given the association of anemia with renal dysfunction, worse hemodynamic conditions, higher NYHA functional class, and longer hospital stay, it is an independent predictor of mortality in decompensated HF [19-21].

One of the main problems to be faced in clinical practice is the absence of early, sensitive, and specific renal function markers for the diagnosis of AKI. Currently, serum creatinine is the most used test to assess renal function, but this 
biomarker changes later in clinical course (after 24 to 48 hours of injury) and, when there is loss of at least $50 \%$ of renal function [22]. Its low accuracy implies late diagnosis and treatment, since a large percentage of individuals who already have acute subclinical kidney injury do not receive timely diagnosis. These patients end up evolving with more severe renal repercussions and increased mortality [23].

Finally, there are promising studies with new biomarkers such as Lipocaine Associated with Neutrophilic Gelatinase (NGAL), which consists of a protein molecule predominantly expressed in the distal part of the nephron after the occurrence of kidney injury. Unlike serum creatinine and glomerular filtration rate, which are markers of renal function, increased levels of NGAL, both in serum and urine, are closely linked to structural nephron lesions [24]. Clinical studies indicate that a few hours after the occurrence of AKI, serum and urinary NGAL levels are already significantly elevated [25]. However, most of the NGAL results described have been obtained through research based on assays using the ELISA method [23], which are not practical in the clinical setting. Thus, due to the lack of scientific and practical validation of such biomarkers so far, renal function remains being assessed by the serum dosage of nitrogenous slags, as this is still the most common method of monitoring renal function and detecting AKI in hospitalized patients.

The main limitation of this study comes from the small sample size and the single-center design. Also, because the study institution is a reference center for the treatment of decompensated $\mathrm{HF}$, results may not reflect the real characteristics of the entire local population. It was not possible to stage AKI according to the AKIN classification, due to the limited data available in medical records, which limited this analysis. Studies with longer clinical follow-up after hospital discharge are necessary, aiming to establish a relationship between parameters of hospitalization and longterm outcomes, such as hospital readmission and mortality.

\section{Conclusion}

The occurrence of AKI was frequent in patients hospitalized due to decompensated HF, occurring in approximately one in five patients. This complication was significantly associated with excess mortality and with the need for IMV during hospitalization, although it was nor associated with increased length of hospital stay. The majority of patients who developed AKI did not have previous CKD.

\section{Data Availability Statement}

Data can be provided by corresponding author Roberto Ramos Barbosa on reasonable request.

\section{Potential Conflict of Interest}

The authors declare that they have no competing interests.

\section{References}

[1] Comitê Coordenador da Diretriz de Insuficiência Cardíaca, Rohde, L., Montera, M. W., Bocchi, E. A., Clausell, N. O., Albuquerque, D. C., et al. (2018). Diretriz Brasileira de Insuficiência Cardíaca Crônica e Aguda. Arquivos Brasileiros de Cardiologia, 111 (3), 436-539.

[2] Núñez, J., Miñana, G., Santas, E., \& Bertomeu-González, V. (2015). Cardiorenal syndrome in acute heart failure: revisiting paradigms. Revista Española de Cardiologia (English Edition), 68 (5), 426-435.

[3] Ponikowski, P., Anker, S. D., AlHabib, K. F., Cowie, M. R., Force, T. L., Hu, S., et al. (2014). Heart failure: preventing disease and death worldwide. ESC heart failure, 1 (1), 4-25.

[4] Greenberg, B. (2012). Acute decompensated heart failure. Circulation Journal, 76 (3), 532-543.

[5] Health Ministry - Sistema de Informações Hospitalares do SUS. SIH/SUS. Available at: http://sihd.datasus.gov.br/principal/index.php.

[6] Ronco, C., Haapio, M., House, A. A., Anavekar, N., \& Bellomo, R. (2008). Cardiorenal syndrome. Journal of the American College of Cardiology, 52 (19), 1527-1539.

[7] Ronco, C., Bellasi, A., \& Di Lullo, L. (2019). Implication of Acute Kidney Injury in Heart Failure. Heart Failure Clinics, 15 (4), 463-476.

[8] Solomon, S. D., Dobson, J., Pocock, S., Skali, H., McMurray, J. J., Granger, C. B., \& Pfeffer, M. A. (2007). Influence of nonfatal hospitalization for heart failure on subsequent mortality in patients with chronic heart failure. Circulation, 116 (13), 1482-7.

[9] Aronson, D., \& Burger, A. J. (2010). The relationship between transient and persistent worsening renal function and mortality in patients with acute decompensated heart failure. Journal of Cardiac Failure, 16 (7), 541-547.

[10] de Bobadilla, J. F., \& López-Sendón, J. (2009). Enfermedad renal: implicaciones terapéuticas en insuficiencia cardíaca y cardiopatía isquémica. Medicina Clínica, 132, 48-54.

[11] Dries, D. L., Exner, D. V., Domanski, M. J., Greenberg, B., \& Stevenson, L. W. (2000). The prognostic implications of renal insufficiency in asymptomatic and symptomatic patients with left ventricular systolic dysfunction. Journal of the American College of Cardiology, 35 (3), 681-689.

[12] Goldberg, R. J., Ismailov, R. M., Patlolla, V., Lessard, D., \& Spencer, F. A. (2008). Therapies for acute heart failure in patients with reduced kidney function: a community-based perspective. American Journal of Kidney Diseases, 51 (4), 594-602.

[13] Heywood, J. T., Fonarow, G. C., Costanzo, M. R., Mathur, V. S., Wigneswaran, J. R., Wynne, J., \& ADHERE Scientific Advisory Committee and Investigators. (2007). High prevalence of renal dysfunction and its impact on outcome in 118,465 patients hospitalized with acute decompensated heart failure: a report from the ADHERE database. Journal of Cardiac Failure, 13 (6), 422-430.

[14] Stauffer, J. L., Olson, D. E., \& Petty, T. L. (1981). Complications and consequences of endotracheal intubation and tracheotomy: a prospective study of 150 critically ill adult patients. The American Journal of Medicine, 70 (1), 65-76. 
[15] Ponce, D., Zorzenon, C. D. P. F., Santos, N. Y. D., Teixeira, U. A., \& Balbi, A. L. (2011). Acute kidney injury in intensive care unit patients: A prospective study on incidence, risk factors and mortality. Revista Brasileira de Terapia Intensiva, 23 (3), 321-326.

[16] Mebazaa, A., Tolppanen, H., Mueller, C., Lassus, J., DiSomma, S., Baksyte, G., et al. (2016). Acute heart failure and cardiogenic shock: a multidisciplinary practical guidance. Intensive care medicine, 42 (2), 147-163.

[17] Garzotto, F., Piccinni, P., Cruz, D., Gramaticopolo, S., Dal Santo, M., Aneloni, G., et al. (2011). RIFLE-based data collection/management system applied to a prospective cohort multicenter Italian study on the epidemiology of acute kidney injury in the intensive care unit. Blood purification, 31 (1-3), 159-171.

[18] Peacock, W. F., Emerman, C., Costanzo, M. R., Diercks, D. B., Lopatin, M., \& Fonarow, G. C. (2009). Early vasoactive drugs improve heart failure outcomes. Congestive Heart Failure, 15 (6), 256-264.

[19] Barbosa, R. R., Storch, T. S., Alves, F. B., Costa, V. S., Nascimento, C. C. R., Torres, L. D., et al. (2019). Hemoglobina e sódio séricos: marcadores prognósticos precoces na insuficiência cardíaca descompensada. Insuf. Card, 2-6.

[20] Reis, F. J. F. B. D., Fernandes, A. M. S., Bitencourt, A. G. V., Neves, F. B. C. S., Kuwano, A. Y., França, V. H. P., et al. (2009). Prevalence of anemia and renal insufficiency in non- hospitalized patients with heart failure. Arquivos Brasileiros de Cardiologia, 93 (3), 268-274.

[21] Sales, A. L. F., Villacorta, H., Reis, L., \& Mesquita, E. T. (2005). Anemia as a prognostic factor in a population hospitalized due to decompensated heart failure. Arquivos Brasileiros de Cardiologia, 84 (3), 237-240.

[22] Peres, L. A. B., Cunha Júnior, A. D. D., Schäfer, A. J., Silva, A L. D., Gaspar, A. D., Scarpari, D. F., et al. (2013). Biomarkers of acute kidney injury. Brazilian Journal of Nephrology, 35 (3), 229-236.

[23] Barros, L. C. N. D., Silveira, F. S., Silveira, M. S., Morais, T. C., Nunes, M. A. P., \& Bastos, K. D. A. (2012). Insuficiência renal aguda em pacientes internados por insuficiência cardíaca descompensada-Reincade. Brazilian Journal of Nephrology, 34 (2), 122-129.

[24] Haase, M., Bellomo, R., Devarajan, P., Schlattmann, P., Haase-Fielitz, A., \& Group, N. M. A. I. (2009). Accuracy of neutrophil gelatinase-associated lipocalin (NGAL) in diagnosis and prognosis in acute kidney injury: a systematic review and meta-analysis. American journal of kidney diseases, 54 (6), 1012-1024.

[25] Schiffl, H., \& Lang, S. M. (2012). Update on biomarkers of acute kidney injury. Molecular diagnosis \& therapy, 16 (4), 199-207. 